\title{
An Efficient Method for Analytically Solving (1+2)- dimensional Chiral Non-linear Schrödinger Equation
}

\section{Muslum Ozisik}

Yildiz Technical University: Yildiz Teknik Universitesi

Mustafa Bayram ( $\sim$ mustafabayram@biruni.edu.tr)

Biruni Universitesi https://orcid.org/0000-0002-2994-7201

\section{Aydin Secer}

Yildiz Technical University: Yildiz Teknik Universitesi

\section{Melih Cinar}

Yildiz Technical University: Yildiz Teknik Universitesi

\section{Research Article}

Keywords: Chiral Nonlinear Schrodinger Equation, soliton solutions, modified extended tanh method combined with new Riccati Solutions

Posted Date: January 3rd, 2022

DOI: https://doi.org/10.21203/rs.3.rs-1142114/v1

License: (a) This work is licensed under a Creative Commons Attribution 4.0 International License. Read Full License 


\title{
An Efficient Method for Analytically Solving (1+2)-dimensional Chiral Non-linear Schrödinger Equation
}

\author{
Muslum Ozisik ${ }^{1}$, Mustafa Bayram ${ }^{2 *}$, Aydin Secer ${ }^{1}$ and Melih \\ Cinar $^{1,3}$
}

${ }^{1}$ Department of Mathematical Engineering, Yildiz Technical

University, Istanbul, 34220, Turkey.

$2^{*}$ Department of Computer Engineering, Istanbul, 34010, Turkey.

${ }^{3}$ Graduate School of Science and Engineering, Yildiz Technical

University, Istanbul, 34220, Turkey.

*Corresponding author(s). E-mail(s):

mustafabayram@biruni.edu.tr;

Contributing authors: ozisik@yildiz.edu.tr; asecer@yildiz.edu.tr;

mcinar@yildiz.edu.tr;

\begin{abstract}
In this paper, we have successfully extracted novel analytic solutions for the (1+2)-dimensional Chiral non-linear Schrödinger (NLS) equation by modified extended tanh expansion method combined with new Riccati solutions (METEM-cNRCS) as far as we know. When a wave transformation is applied to the considered Chiral NLS equation, a nonlinear ODE is obtained. Assuming the solutions of ODE have a form as the method suggests, and substituting the trial solutions to the ODE, we get a polynomial. Gathering the coefficients with the same power in the polynomial, we acquire an algebraic equation system. So, we may obtain the abundant solutions of the $(1+2)$-dimensional Chiral NLS equation by solving the system via Maple. The plots of some solutions are demonstrated to explain the dynamics of the solutions. It is expected that the results of the paper are a guide for future works in traveling wave theory.
\end{abstract}

Keywords: Chiral Nonlinear Schrodinger Equation, soliton solutions, modified extended tanh method combined with new Riccati Solutions. 


\section{Introduction}

Researchers can best model real-world phenomena in various fields using partial differential equations (PDEs). Since the real-world phenomena show non-linear behavior, the non-linear terms are also frequently observed in the PDEs. So, we often come across non-linear PDEs in natural sciences such as physics, chemistry, and biology [1-11]. Besides, PDEs have recently been used in new areas such as machine learning and microbiology, etc. On the other hand, some complicated phenomena might be best modeled using non-linear Schrödinger equations (NLSE). Some of the studies on NLSE are topological behavior of Schrödinger equation in Besov spaces [12], Darboux transformation [13] , statistical mechanics of Schrödinger equation in complex fields [14], relaxation scheme [15], modification for deep waves [16], nonautonomous complex wave solutions of NLSE [17], inverse scattering transformation [18], thirring solutions of NLSE model birefringent fibers [19], dissipative nonlinear Schrödinger equation [20], differential quadrature algorithm [21] and modulation instability analysis [22], etc.

The concept of the solitons might appears in every moment of life. Optical solitons, one of the soliton types, play a vital role in physics and telecommunication engineering. So, it is attractive topic for many researchers. The solitons of the Chiral NLS equation are extremely important in the quantum Hall effects that are one of fundamentals in nonlinear optics. In the literature, some exact solutions of Chiral NLS equation have been derived via different methods such as modified Khater and modified Jacobian expansion methods [23], the soliton ansatz method [24], the modified Jacobi elliptic expansion method [25], trial solution method [26]. But this study aims to derive new soliton solutions of the considered equation. The considered method is so fresh and produces abundant analytical solutions for non-linear PDEs in various science areas.

In this paper, we have interested in finding the new exact solutions of the following (1+2)-dimensional Chiral NLS equation [24, 27] by METEM-cNRCS [28]:

$i \frac{\partial u}{\partial t}+\beta\left(\frac{\partial^{2} u}{\partial x^{2}}+\frac{\partial^{2} u}{\partial y^{2}}\right)+i\left(\alpha_{1}\left(u \frac{\partial u^{*}}{\partial x}-u^{*} \frac{\partial u}{\partial x}\right)+\alpha_{2}\left(u \frac{\partial u^{*}}{\partial y}-u^{*} \frac{\partial u}{\partial y}\right)\right) u=0$,

where $i=\sqrt{-1}, u=u(x, y, t)$ is a complex function, the first term is the evolution term, $\alpha_{1}, \alpha_{2}$ are the coefficients of nonlinear coupling terms, $\beta$ denotes the dispersion coefficient, and * represents complex conjugate.

The rest of the work will continue as follows: we give an explanation of the method in section 2. METEM-cNRCS is applied to $(1+2)$-dimensional Chiral NLS equation in section 3. Section 4 interprets the results of the study and the plots of the constructed solutions. The final chapter includes the conclusion of the paper. 


\section{Method}

In this section, a brief for the METEM-cNRCS is explained.

Step 1: Let's deal with the NLPDE in the general form and the following wave transformations, respectively:

$$
\begin{gathered}
F\left(\chi, \frac{\partial \chi}{\partial t}, \frac{\partial \chi}{\partial x}, \frac{\partial^{2} \chi}{\partial t^{2}}, \frac{\partial^{2} \chi}{\partial x^{2}}, \frac{\partial^{2} \chi}{\partial x \partial t}, \ldots\right)=0 \\
u(x, y, t)=e^{i \theta} \chi(\delta), \quad \delta=p_{1} x+p_{2} y+p_{3} t, \quad \theta=\lambda_{1} x+\lambda_{2} y+\omega t+\varphi .
\end{gathered}
$$

After we substitute Eq. 3 to Eq. 2, we get:

$$
P\left(\chi(\delta), \chi^{\prime}(\delta), \chi^{\prime \prime}(\delta), \ldots\right)=0 .
$$

where it is the general of an ODE and the prime symbolizes the derivative of $\chi(\delta)$ with respect to $\delta$.

Step 2: Assume that Eq. 4 has the solutions in the form:

$$
\chi(\delta)=A_{0}+\sum_{i=1}^{m} A_{i} \Phi^{i}(\delta)+\sum_{i=1}^{m} B_{i} \Phi^{-i}(\delta), \quad A_{m}, B_{m} \neq 0 .
$$

where $A_{0}, A_{1}, \ldots, A_{m}$ and $B_{1}, \ldots, B_{m}$ are real numbers to be found ( $A_{m}$ and $B_{m}$ should not be zero, simultaneously) and $m$ is an integer that can be determined by balancing the terms with highest derivative term and the highest power non-linear term in Eq. 4. Additionally, $\Phi(\delta)$ is a solution of Riccati differential equation:

$$
\frac{d \Phi(\delta)}{d \delta}=\rho+[\Phi(\delta)]^{2}
$$

in which $\rho$ is a real constant.

Step 3: Use the new exact solutions of Eq. 6 which are given in Table 1. It is the main difference between the modified extended tanh method and METEM-cNRCS.

Table 1 The new exact solutions of Riccati equation in Eq. 6 with $\mu=\mp 1$ [29].

\begin{tabular}{|c|c|}
\hline$\Phi(\delta)$ for $\rho<0$ & $\Phi(\delta)$ for $\rho>0$ \\
\hline$\Phi_{1}(\delta)=-\sqrt{-\rho}(\tanh (2 \sqrt{-\rho} \delta)+i \mu \operatorname{sech}(2 \sqrt{-\rho} \delta))$ & $\Phi_{7}(\delta)=\sqrt{\rho}(\tan (2 \sqrt{\rho} \delta)+\mu \sec (2 \sqrt{\rho} \delta))$ \\
\hline$\Phi_{2}(\delta)=-\sqrt{-\rho}(\operatorname{coth}(2 \sqrt{-\rho} \delta)+\mu \operatorname{csch}(2 \sqrt{-\rho} \delta))$ & $\Phi_{8}(\delta)=-\sqrt{\rho}(\cot (2 \sqrt{\rho} \delta)+\mu \csc (2 \sqrt{\rho} \delta))$ \\
\hline$\Phi_{3}(\delta)=-\frac{1}{2} \sqrt{-\rho}\left(\tanh \left(\frac{\sqrt{-\rho}}{2} \delta\right)+\operatorname{coth}\left(\frac{\sqrt{-\rho}}{2} \delta\right)\right)$ & $\Phi_{9}(\delta)=\frac{1}{2} \sqrt{\rho}\left(\tan \left(\frac{\sqrt{\rho}}{2} \delta\right)-\cot \left(\frac{\sqrt{\rho}}{2} \delta\right)\right)$ \\
\hline$\Phi_{4}(\delta)=\frac{\rho-\sqrt{-\rho} \tanh (\sqrt{-\rho} \delta)}{1+\sqrt{-\rho} \tanh (\sqrt{-\rho} \delta)}$ & $\Phi_{10}(\delta)=\frac{-\sqrt{\rho}(1-\tan (\sqrt{\rho} \delta))}{1+\tan (\sqrt{\rho} \delta)}$ \\
\hline$\Phi_{5}(\delta)=\frac{\sqrt{-\rho}(5-4 \cosh (2 \sqrt{-\rho} \delta))}{3+4 \sinh (2 \sqrt{-\rho} \delta)}$ & $\Phi_{11}(\delta)=\frac{\sqrt{\rho}(4-5 \cos (2 \sqrt{\rho} \delta))}{3+5 \sin (2 \sqrt{\rho} \delta)}$ \\
\hline$\Phi_{6}(\delta)=\frac{-2 \sqrt{-\rho}}{\tanh \left(\frac{\sqrt{-\rho}}{2} \delta\right)+\operatorname{coth}\left(\frac{\sqrt{-\rho}}{2} \delta\right)}$ & $\Phi_{12}(\delta)=\frac{\tan \left(\frac{\sqrt{\rho}}{2} \delta\right)-\cot \left(\frac{\sqrt{\rho}}{2} \delta\right)}{}$ \\
\hline
\end{tabular}


Step 4: Substitute Eq. 5 and its derivatives to Eq. 4 and using Eq. 6, we attain a polynomial in $\Phi(\delta)$. When we gather the coefficients of $\Phi(\delta)$ including the same power and equate each coefficient to zero, we have a system to be solved. Step 5: Find the unknowns $A_{0}, A_{1}, \ldots, A_{m}, B_{1}, \ldots, B_{m}, p_{1}, p_{2}, p_{3}, \lambda_{1}, \lambda_{2}, \lambda_{3}, \omega$ and $\varphi$ by solving the system in Step 4 . The solutions of the main equation is extracted by substituting the unknowns into Eq. 5 and using Eq. 3.

\section{Application}

In this section, we will apply our method to $(1+2)$-dimensional Chiral NLS equation in Eq. 1. We will first use the following wave transformations in order to convert the NLS equation to an ODE:

$$
u(x, y, t)=\chi(\delta) e^{i \theta}, \quad \delta=b_{1} x+b_{2} y-v t, \quad \theta=\lambda_{1} x+\lambda_{2} y+\omega t+\varphi
$$

After the transformation, the following equations can be deduced from the imaginary and the real parts:

$$
\begin{gathered}
\left(2 \beta b_{1} \lambda_{1}+2 \beta b_{2} \lambda_{2}-v\right) \frac{\mathrm{d}}{\mathrm{d} \delta} \chi(\delta)=0 \\
\left(2 \alpha_{1} \lambda_{1}+2 \alpha_{2} \lambda_{2}\right)(\chi(\delta))^{3}+\left(-\beta{\lambda_{1}}^{2}-\beta{\lambda_{2}}^{2}-\omega\right) \chi(\delta) \\
+\left(\beta{b_{1}}^{2}+\beta{b_{2}}^{2}\right) \frac{\mathrm{d}^{2}}{\mathrm{~d} \delta^{2}} \chi(\delta)=0 .
\end{gathered}
$$

Besides, one might get from Eq. 8:

$$
v=2 \beta\left(b_{1} \lambda_{1}+b_{2} \lambda_{2}\right)
$$

We get the ODE form from Eq. 9:

$2\left(\alpha_{1} \lambda_{1}+\alpha_{2} \lambda_{2}\right) \chi^{3}(\delta)+\left(-\beta{\lambda_{1}}^{2}-\beta{\lambda_{2}}^{2}-\omega\right) \chi(\delta)+\left(\beta b_{1}{ }^{2}+\beta b_{2}{ }^{2}\right) \chi^{\prime \prime}(\delta)=0$,

Balancing $\chi^{\prime \prime}$ and $\chi^{3}$ in Eq. 11 , we get $m+2=3 m$, so $m=1$. According to the considered method, the solutions of Eq. 11 can be assumed to be following series form:

$$
\chi(\delta)=A_{0}+A_{1} \Phi(\delta)+B_{1} \frac{1}{\Phi(\delta)}, \quad A_{1}, B_{1} \neq 0,
$$

where $A_{1}$ and $B_{1}$ should not be zero, simultaneously. When the Eq. 12 and its related derivatives are substituted to Eq. 11, a polynomial in $\Phi(\delta)$ are derived by considering Eq. 6 . Collecting the terms with the same power of $\Phi^{i}(\delta)$ and equating the all coefficients to 0 , we attain a system: 


$$
\begin{aligned}
& \Phi^{-3}(\delta): \quad 2\left(\left(\alpha_{1} \lambda_{1}+\alpha_{2} \lambda_{2}\right) B_{1}^{2}+\rho^{2} \beta\left(b_{1}{ }^{2}+b_{2}{ }^{2}\right)\right) B_{1}=0, \\
& \Phi^{-2}(\delta): \quad 6 A_{0} B_{1}^{2}\left(\alpha_{1} \lambda_{1}+\alpha_{2} \lambda_{2}\right)=0, \\
& \Phi^{-1}(\delta): 2 B_{1}\left(\left(-1 / 2{\lambda_{1}}^{2}-1 / 2{\lambda_{2}}^{2}+\rho\left(b_{1}{ }^{2}+b_{2}{ }^{2}\right)\right) \beta\right. \\
& \left.+3 \alpha_{1}\left(A_{0}^{2}+A_{1} B_{1}\right) \lambda_{1}+3 \alpha_{2}\left(A_{0}^{2}+A_{1} B_{1}\right) \lambda_{2}-\omega / 2\right)=0 \text {, } \\
& \Phi^{0}(\delta): \quad-\left(\beta \lambda_{1}{ }^{2}-2 \alpha_{1}\left(A_{0}{ }^{2}+6 A_{1} B_{1}\right) \lambda_{1}+\beta \lambda_{2}{ }^{2}\right. \\
& \left.-2 \alpha_{2}\left(A_{0}^{2}+6 A_{1} B_{1}\right) \lambda_{2}+\omega\right) A_{0}=0 \text {, } \\
& \Phi^{1}(\delta): 2 A_{1}\left(\left(-1 / 2{\lambda_{1}}^{2}-1 / 2{\lambda_{2}}^{2}+\rho\left(b_{1}{ }^{2}+b_{2}{ }^{2}\right)\right) \beta\right. \\
& \left.+3 \alpha_{1}\left(A_{0}^{2}+A_{1} B_{1}\right) \lambda_{1}+3 \alpha_{2}\left(A_{0}^{2}+A_{1} B_{1}\right) \lambda_{2}-\omega / 2\right)=0 \text {, } \\
& \Phi^{2}(\delta): \quad 6 A_{0} A_{1}^{2}\left(\alpha_{1} \lambda_{1}+\alpha_{2} \lambda_{2}\right)=0, \\
& \Phi^{3}(\delta): \quad 2 A_{1}{ }^{3} \alpha_{1} \lambda_{1}+2 A_{1}{ }^{3} \alpha_{2} \lambda_{2}+2 A_{1} \beta b_{1}{ }^{2}+2 A_{1} \beta b_{2}{ }^{2}=0 .
\end{aligned}
$$

The unknowns $A_{0}, A_{1}, B_{1}, \alpha, k, p, a, b, w$ and $\sigma$ can be determined solving the system by Maple. The possible 36 cases for the solutions of the system are added to Appendix. Using the $\Phi_{i}(\delta)$ in Table 1 and all cases in Appendix, we obtain the following solutions for NLPDE in Eq. 1.

$$
\begin{gathered}
\Phi_{1}(\delta)=-\sqrt{-\rho}(\tanh (2 \sqrt{-\rho} \delta)+i \operatorname{sech}(2 \sqrt{-\rho} \delta)), \\
u_{1}(x, y, t)=\Upsilon_{1}\left(\begin{array}{l}
A_{0}-A_{1} \sqrt{-\rho}\left(\begin{array}{c}
\tanh \left(2 \sqrt{-\rho}\left(b_{1} x+b_{2} y-\left(2 \beta b_{1} \lambda_{1}+2 \beta b_{2} \lambda_{2}\right) t\right)\right) \\
+i \operatorname{sech}\left(2 \sqrt{-\rho}\left(b_{1} x+b_{2} y-\left(2 \beta b_{1} \lambda_{1}+2 \beta b_{2} \lambda_{2}\right) t\right)\right)
\end{array}\right) \\
-B_{1} \frac{1}{\sqrt{-\rho}}\left(\begin{array}{c}
\tanh \left(2 \sqrt{-\rho}\left(b_{1} x+b_{2} y-\left(2 \beta b_{1} \lambda_{1}+2 \beta b_{2} \lambda_{2}\right) t\right)\right) \\
+i \operatorname{sech}\left(2 \sqrt{-\rho}\left(b_{1} x+b_{2} y-\left(2 \beta b_{1} \lambda_{1}+2 \beta b_{2} \lambda_{2}\right) t\right)\right)
\end{array}\right)
\end{array}\right),
\end{gathered}
$$

where $\Upsilon_{1}=e^{i\left(\omega t+\lambda_{1} x+\lambda_{2} y+\varphi\right)}$

$$
\begin{aligned}
& \Phi_{2}(\delta)=-\sqrt{-\rho}(\operatorname{coth}(2 \sqrt{-\rho} \delta)+\mu \operatorname{csch}(2 \sqrt{-\rho} \delta)) \\
& u_{2}(x, y, t)=\Upsilon_{1}\left(\begin{array}{c}
A_{0}-A_{1} \sqrt{-\rho}\left(\begin{array}{c}
\operatorname{coth}\left(2 \sqrt{-\rho}\left(b_{1} x+b_{2} y-\left(2 \beta b_{1} \lambda_{1}+2 \beta b_{2} \lambda_{2}\right) t\right)\right) \\
+\operatorname{csch}\left(2 \sqrt{-\rho}\left(b_{1} x+b_{2} y-\left(2 \beta b_{1} \lambda_{1}+2 \beta b_{2} \lambda_{2}\right) t\right)\right)
\end{array}\right) \\
-B_{1} \frac{1}{\sqrt{-\rho}}\left(\begin{array}{c}
\operatorname{coth}\left(2 \sqrt{-\rho}\left(b_{1} x+b_{2} y-\left(2 \beta b_{1} \lambda_{1}+2 \beta b_{2} \lambda_{2}\right) t\right)\right) \\
+\operatorname{csch}\left(2 \sqrt{-\rho}\left(b_{1} x+b_{2} y-\left(2 \beta b_{1} \lambda_{1}+2 \beta b_{2} \lambda_{2}\right) t\right)\right)
\end{array}\right)
\end{array}\right), \\
& \Phi_{3}(\delta)=-\frac{1}{2} \sqrt{-\rho}\left(\tanh \left(\frac{\sqrt{-\rho}}{2} \delta\right)+\operatorname{coth}\left(\frac{\sqrt{-\rho}}{2} \delta\right)\right), \\
& u_{3}(x, y, t)=\Upsilon_{1}\left(\begin{array}{c}
A_{0}-\frac{A_{1}}{2} \sqrt{-\rho}\left(\begin{array}{l}
\tanh \left(\frac{b_{1} x+b_{2} y-\left(2 \beta b_{1} \lambda_{1}+2 \beta b_{2} \lambda_{2}\right) t}{2} \sqrt{-\rho}\right) \\
+\operatorname{coth}\left(\frac{b_{1} x+b_{2} y-\left(2 \beta b_{1} \lambda_{1}+2 \beta b_{2} \lambda_{2}\right) t}{2} \sqrt{-\rho}\right)
\end{array}\right) \\
-2 \frac{B_{1}}{\sqrt{-\rho}\left(\begin{array}{c}
\tanh \left(1 / 2 \sqrt{-\rho}\left(b_{1} x+b_{2} y-\left(2 \beta b_{1} \lambda_{1}+2 \beta b_{2} \lambda_{2}\right) t\right)\right) \\
+\operatorname{coth}\left(1 / 2 \sqrt{-\rho}\left(b_{1} x+b_{2} y-\left(2 \beta b_{1} \lambda_{1}+2 \beta b_{2} \lambda_{2}\right) t\right)\right)
\end{array}\right)}
\end{array}\right), \\
& \Phi_{4}(\delta)=(\rho-\sqrt{-\rho} \tanh (\sqrt{-\rho} \delta))(1+\sqrt{-\rho} \tanh (\sqrt{-\rho} \delta))^{-1}
\end{aligned}
$$




$$
\begin{aligned}
& u_{4}(x, y, t)=\Upsilon_{1}\left(\begin{array}{l}
A_{0}+A_{1}\left(\rho-\sqrt{-\rho} \tanh \left(\sqrt{-\rho}\left(b_{1} x+b_{2} y-\left(2 \beta b_{1} \lambda_{1}+2 \beta b_{2} \lambda_{2}\right) t\right)\right)\right) \\
\left(1+\sqrt{-\rho} \tanh \left(\sqrt{-\rho}\left(b_{1} x+b_{2} y-\left(2 \beta b_{1} \lambda_{1}+2 \beta b_{2} \lambda_{2}\right) t\right)\right)\right)^{-1} \\
+B_{1}\left(1+\sqrt{-\rho} \tanh \left(\sqrt{-\rho}\left(b_{1} x+b_{2} y-\left(2 \beta b_{1} \lambda_{1}+2 \beta b_{2} \lambda_{2}\right) t\right)\right)\right) \\
\left(\rho-\sqrt{-\rho} \tanh \left(\sqrt{-\rho}\left(b_{1} x+b_{2} y-\left(2 \beta b_{1} \lambda_{1}+2 \beta b_{2} \lambda_{2}\right) t\right)\right)\right)^{-1}
\end{array}\right), \\
& \Phi_{5}(\delta)=\sqrt{-\rho}(5-4 \cosh (2 \sqrt{-\rho} \delta))(3+4 \sinh (2 \sqrt{-\rho} \delta))^{-1} \\
& u_{5}(x, y, t)=\Upsilon_{1}\left(\begin{array}{l}
A_{0}+A_{1} \sqrt{-\rho}\left(5-4 \cosh \left(2 \sqrt{-\rho}\left(b_{1} x+b_{2} y-\left(2 \beta b_{1} \lambda_{1}+2 \beta b_{2} \lambda_{2}\right) t\right)\right)\right) \\
\left(3+4 \sinh \left(2 \sqrt{-\rho}\left(b_{1} x+b_{2} y-\left(2 \beta b_{1} \lambda_{1}+2 \beta b_{2} \lambda_{2}\right) t\right)\right)\right)^{-1} \\
+B_{1}\left(3+4 \sinh \left(2 \sqrt{-\rho}\left(b_{1} x+b_{2} y-\left(2 \beta b_{1} \lambda_{1}+2 \beta b_{2} \lambda_{2}\right) t\right)\right)\right) \\
\frac{1}{\sqrt{-\rho}}\left(5-4 \cosh \left(2 \sqrt{-\rho}\left(b_{1} x+b_{2} y-\left(2 \beta b_{1} \lambda_{1}+2 \beta b_{2} \lambda_{2}\right) t\right)\right)\right)^{-1}
\end{array}\right), \\
& \Phi_{6}(\delta)=-2 \sqrt{-\rho}\left(\tanh \left(\frac{\sqrt{-\rho}}{2} \delta\right)+\operatorname{coth}\left(\frac{\sqrt{-\rho}}{2} \delta\right)\right)^{-1} \\
& u_{6}(x, y, t)=\Upsilon_{1}\left(\begin{array}{c}
A_{0}-2 \frac{A_{1} \sqrt{-\rho}}{\tanh \left(1 / 2 \sqrt{-\rho}\left(b_{1} x+b_{2} y-\left(2 \beta b_{1} \lambda_{1}+2 \beta b_{2} \lambda_{2}\right) t\right)\right)} \\
+\operatorname{coth}\left(1 / 2 \sqrt{-\rho}\left(b_{1} x+b_{2} y-\left(2 \beta b_{1} \lambda_{1}+2 \beta b_{2} \lambda_{2}\right) t\right)\right) \\
-\frac{B_{1}}{2} \frac{1}{\sqrt{-\rho}}\left(\begin{array}{l}
\tanh \left(\frac{b_{1} x+b_{2} y-\left(2 \beta b_{1} \lambda_{1}+2 \beta b_{2} \lambda_{2}\right) t}{2} \sqrt{-\rho}\right) \\
+\operatorname{coth}\left(\frac{b_{1} x+b_{2} y-\left(2 \beta b_{1} \lambda_{1}+2 \beta b_{2} \lambda_{2}\right) t}{2} \sqrt{-\rho}\right)
\end{array}\right)
\end{array}\right), \\
& \Phi_{7}(\delta)=\sqrt{\rho}(\tan (2 \sqrt{\rho} \delta)+\mu \sec (2 \sqrt{\rho} \delta)) \\
& u_{7}(x, y, t)=\Upsilon_{1}\left(\begin{array}{c}
A_{0}+A_{1} \sqrt{\rho}\left(\begin{array}{c}
\tan \left(2 \sqrt{\rho}\left(b_{1} x+b_{2} y-\left(2 \beta b_{1} \lambda_{1}+2 \beta b_{2} \lambda_{2}\right) t\right)\right) \\
+\sec \left(2 \sqrt{\rho}\left(b_{1} x+b_{2} y-\left(2 \beta b_{1} \lambda_{1}+2 \beta b_{2} \lambda_{2}\right) t\right)\right)
\end{array}\right) \\
+B_{1} \frac{1}{\sqrt{\rho}}\left(\begin{array}{l}
\tan \left(2 \sqrt{\rho}\left(b_{1} x+b_{2} y-\left(2 \beta b_{1} \lambda_{1}+2 \beta b_{2} \lambda_{2}\right) t\right)\right) \\
+\sec \left(2 \sqrt{\rho}\left(b_{1} x+b_{2} y-\left(2 \beta b_{1} \lambda_{1}+2 \beta b_{2} \lambda_{2}\right) t\right)\right)
\end{array}\right)
\end{array}\right), \\
& \Phi_{8}(\delta)=-\sqrt{\rho}(\cot (2 \sqrt{f} \delta)+\mu \csc (2 \sqrt{\rho} \delta)) \\
& u_{8}(x, y, t)=\Upsilon_{1}\left(\begin{array}{c}
A_{0}-A_{1} \sqrt{\rho}\left(\begin{array}{c}
\cot \left(2 \sqrt{\rho}\left(b_{1} x+b_{2} y-\left(2 \beta b_{1} \lambda_{1}+2 \beta b_{2} \lambda_{2}\right) t\right)\right) \\
+\csc \left(2 \sqrt{\rho}\left(b_{1} x+b_{2} y-\left(2 \beta b_{1} \lambda_{1}+2 \beta b_{2} \lambda_{2}\right) t\right)\right)
\end{array}\right) \\
-B_{1} \frac{1}{\sqrt{\rho}}\left(\begin{array}{c}
\cot \left(2 \sqrt{\rho}\left(b_{1} x+b_{2} y-\left(2 \beta b_{1} \lambda_{1}+2 \beta b_{2} \lambda_{2}\right) t\right)\right) \\
+\csc \left(2 \sqrt{\rho}\left(b_{1} x+b_{2} y-\left(2 \beta b_{1} \lambda_{1}+2 \beta b_{2} \lambda_{2}\right) t\right)\right)
\end{array}\right)
\end{array}\right), \\
& \Phi_{9}(\eta)=\frac{1}{2} \sqrt{\rho}\left(\tan \left(\frac{\sqrt{\rho}}{2} \eta\right)-\cot \left(\frac{\sqrt{\rho}}{2} \eta\right)\right) \\
& u_{9}(x, y, t)=\Upsilon_{1}\left(\begin{array}{c}
A_{0}+\frac{A_{1}}{2} \sqrt{\rho}\left(\begin{array}{l}
\tan \left(\frac{b_{1} x+b_{2} y-\left(2 \beta b_{1} \lambda_{1}+2 \beta b_{2} \lambda_{2}\right) t}{2} \sqrt{\rho}\right) \\
-\cot \left(\frac{b_{1} x+b_{2} y-\left(2 \beta b_{1} \lambda_{1}+2 \beta b_{2} \lambda_{2}\right) t}{2} \sqrt{\rho}\right)
\end{array}\right) \\
+2 \frac{B_{1}}{\sqrt{\rho}\left(\begin{array}{l}
\tan \left(1 / 2 \sqrt{\rho}\left(b_{1} x+b_{2} y-\left(2 \beta b_{1} \lambda_{1}+2 \beta b_{2} \lambda_{2}\right) t\right)\right) \\
-\cot \left(1 / 2 \sqrt{\rho}\left(b_{1} x+b_{2} y-\left(2 \beta b_{1} \lambda_{1}+2 \beta b_{2} \lambda_{2}\right) t\right)\right)
\end{array}\right)}
\end{array}\right), \\
& \Phi_{10}(\delta)=-\sqrt{\rho}(1-\tan (\sqrt{\rho} \delta))(1+\tan (\sqrt{\rho} \delta))^{-1}
\end{aligned}
$$




$$
\left.\begin{array}{c}
u_{10}(x, y, t)=\Upsilon_{1}\left(\begin{array}{l}
A_{0}-A_{1} \sqrt{\rho}\left(1-\tan \left(\sqrt{\rho}\left(b_{1} x+b_{2} y-\left(2 \beta b_{1} \lambda_{1}+2 \beta b_{2} \lambda_{2}\right) t\right)\right)\right) \\
\left(1+\tan \left(\sqrt{\rho}\left(b_{1} x+b_{2} y-\left(2 \beta b_{1} \lambda_{1}+2 \beta b_{2} \lambda_{2}\right) t\right)\right)\right)^{-1} \\
-B_{1}\left(1+\tan \left(\sqrt{\rho}\left(b_{1} x+b_{2} y-\left(2 \beta b_{1} \lambda_{1}+2 \beta b_{2} \lambda_{2}\right) t\right)\right)\right) \\
\frac{1}{\sqrt{\rho}}\left(1-\tan \left(\sqrt{\rho}\left(b_{1} x+b_{2} y-\left(2 \beta b_{1} \lambda_{1}+2 \beta b_{2} \lambda_{2}\right) t\right)\right)\right)^{-1}
\end{array}\right), \\
\Phi_{11}(\delta)=\sqrt{\rho}(4-5 \cos (2 \sqrt{\rho} \delta))(3+5 \sin (2 \sqrt{\rho} \delta))^{-1}, \\
u_{11}(x, y, t)=\Upsilon_{1}\left(\begin{array}{l}
A_{0}+A_{1} \sqrt{\rho}\left(4-5 \cos \left(2 \sqrt{\rho}\left(b_{1} x+b_{2} y-\left(2 \beta b_{1} \lambda_{1}+2 \beta b_{2} \lambda_{2}\right) t\right)\right)\right) \\
\left(3+5 \sin \left(2 \sqrt{\rho}\left(b_{1} x+b_{2} y-\left(2 \beta b_{1} \lambda_{1}+2 \beta b_{2} \lambda_{2}\right) t\right)\right)\right)^{-1} \\
+B_{1}\left(3+5 \sin \left(2 \sqrt{\rho}\left(b_{1} x+b_{2} y-\left(2 \beta b_{1} \lambda_{1}+2 \beta b_{2} \lambda_{2}\right) t\right)\right)\right) \\
\frac{1}{\sqrt{\rho}}\left(4-5 \cos \left(2 \sqrt{\rho}\left(b_{1} x+b_{2} y-\left(2 \beta b_{1} \lambda_{1}+2 \beta b_{2} \lambda_{2}\right) t\right)\right)\right)^{-1}
\end{array}\right.
\end{array}\right),
$$

\section{Results and discussion}

Even though there are many methods in the literature and also the considered equation were solved via many methods, to our best knowledge we have derived some new exact solutions of the $(1+2)$ Chiral NLS equation. The METEMcNRCS has been used for the Chiral NLS equation. To fulfill the algorithm of the method, all computations have been done by Maple. Moreover, two and three-dimensional plots of some solutions have been given to demonstrate how the solutions of the considered equation behave. The results of this study are expected to have a guide for further study in optical physics.

For example, we have plotted the 3D plots of the modulus (Fig.1-a), real part (Fig.1-b), imaginary part (Fig.1-c), and 2D plots of modulus (Fig.1-d), real part (Fig.1-e), imaginary part (Fig.1-f) and contour plots of modulus (Fig.1-g), real part (Fig.1-h) and imaginary part (Fig.1-i) of $u_{1}(x, y, t)$ by considering Set $_{1}$ in Eq. 1 for the parameters $\rho=-0.4, A_{1}=b_{1}=b_{2}=\alpha_{1}=\alpha_{2}=$ $\lambda_{2}=\varphi=t=1, \beta=-1$. Besides, some of the other obtained solutions are similarly plotted in the Figures 2-6. We expect that the novel solutions and outcomes of the study will be inspiration for further works in different fields in non-linear science. 


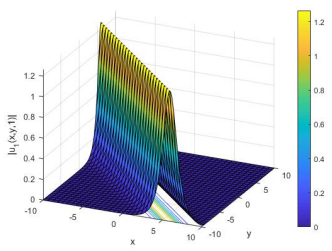

(a) 3D modulus,

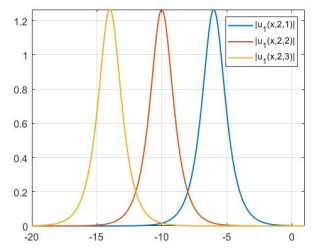

(d) 2D modulus,

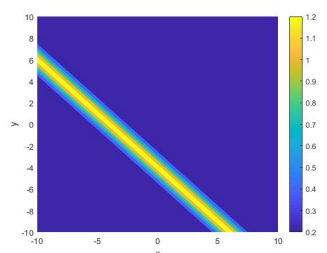

(g) Contour of the modulus,

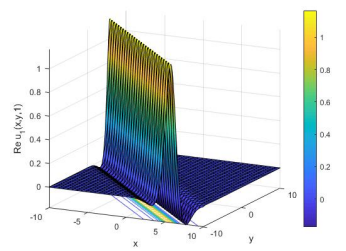

(b) 3D real,

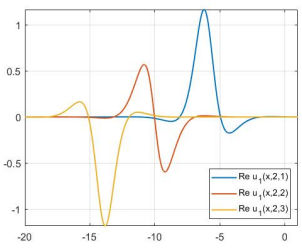

(e) 2D real,

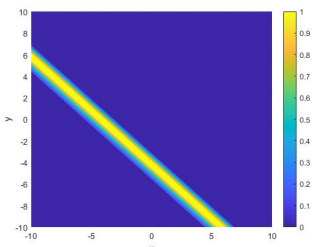

(h) Contour of the real,

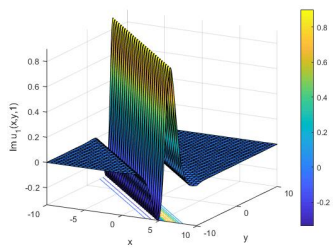

(c) 3D imaginary,

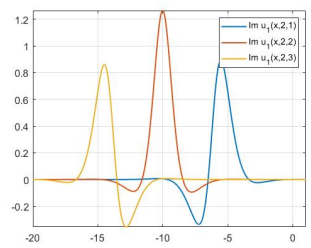

(f) 2D imaginary,

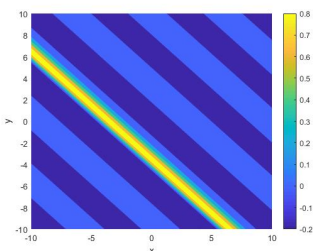

(i) Contour of the imaginary,

Fig. 1 Plots of the solution $u_{1}(x, y, t)$ in Eq. 14 for the parameters $\rho=-0.4, A_{1}=b_{1}=$ $b_{2}=\alpha_{1}=\alpha_{2}=\lambda_{2}=\varphi=t=1, \beta=-1$, and Set $_{1}$ 


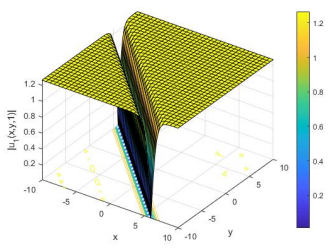

(a) 3D modulus,

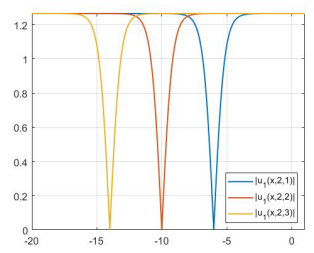

(d) 2D modulus,

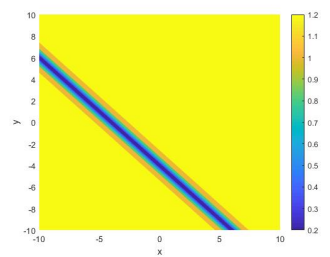

(g) Contour of the modulus,

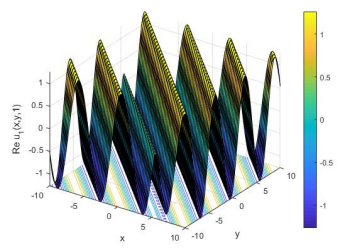

(b) 3D real,

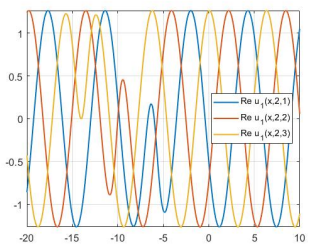

(e) $2 \mathrm{D}$ real,

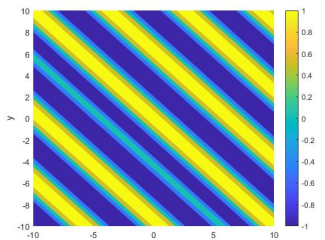

(h) Contour of the real,

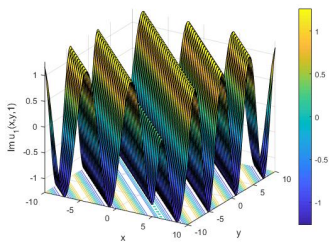

(c) 3D imaginary,

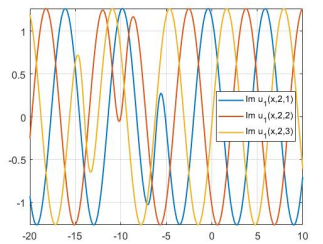

(f) $2 \mathrm{D}$ imaginary,

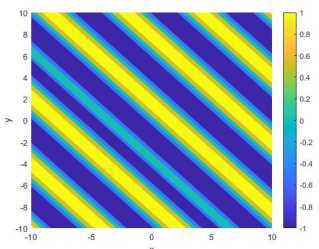

(i) Contour of the imaginary,

Fig. 2 Plots of the solution $u_{1}(x, y, t)$ in Eq. 21 for the parameters $\rho=-0.4, A_{1}=b_{1}=$ $b_{2}=\alpha_{1}=\alpha_{2}=\lambda_{2}=\varphi=t=1, \beta=-1$ and Set $_{2}$ 


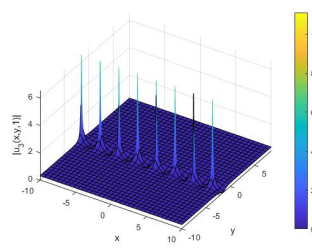

(a) 3D modulus,

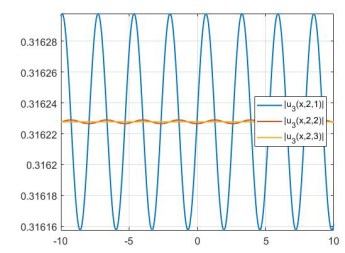

(d) 2D modulus,

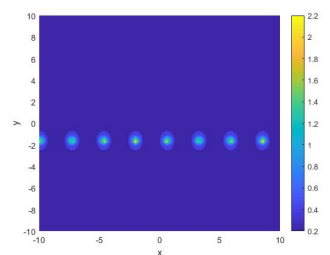

(g) Contour of the modulus,

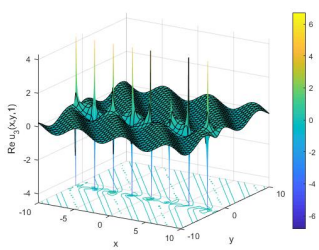

(b) 3D real,

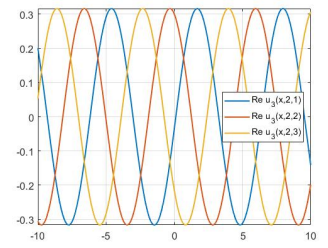

(e) $2 \mathrm{D}$ real,

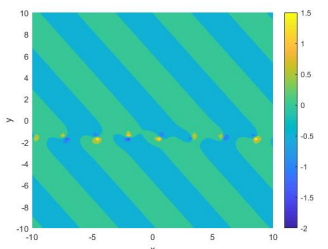

(h) Contour of the real,

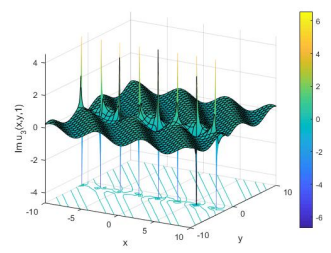

(c) 3D imaginary,

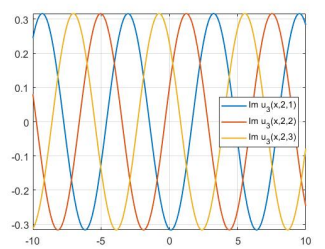

(f) $2 \mathrm{D}$ imaginary,

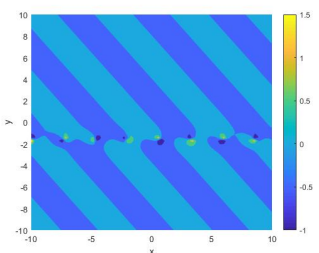

(i) Contour of the imaginary,

Fig. 3 Plots of the solution $u_{3}(x, y, t)$ in Eq. 14 for the parameters $\rho=-0.4, b_{2}=\alpha_{1}=$ $\alpha_{2}=\lambda_{1}=\varphi=t=1, \beta=-1, \lambda_{2}=0.8, \omega=2$, and Set $_{22}$ 


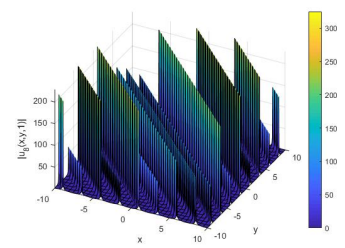

(a) 3D modulus,

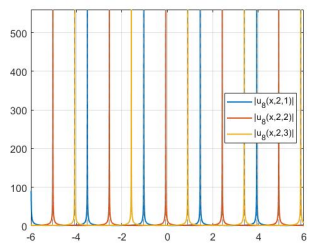

(d) 2D modulus,

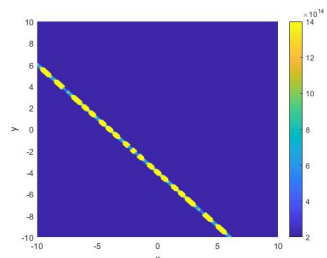

(g) Contour of the modulus,

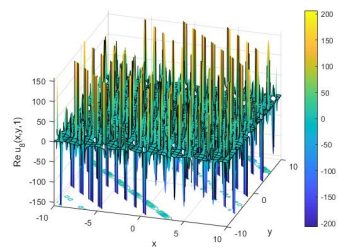

(b) 3D real,

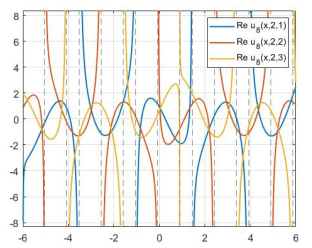

(e) $2 \mathrm{D}$ real,

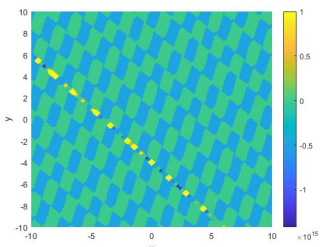

(h) Contour of the real,

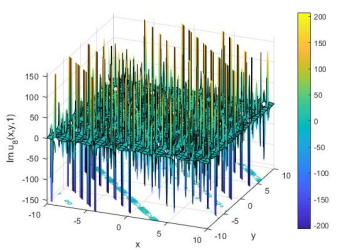

(c) 3D imaginary,

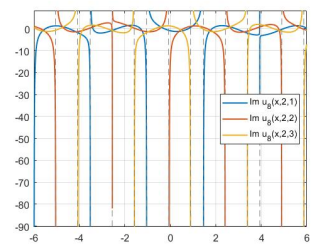

(f) $2 \mathrm{D}$ imaginary,

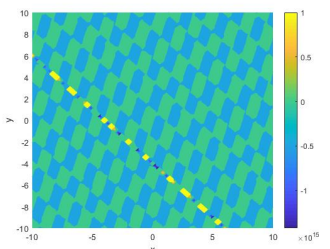

(i) Contour of the imaginary,

Fig. 4 Plots of the solution $u_{8}(x, y, t)$ in Eq. 14 for the parameters $\rho=0.4, A_{1}=b_{1}=b_{2}=$ $\alpha_{1}=\alpha_{2}=\beta=\lambda_{2}=\varphi=t=1$, and Set $_{1}$ 


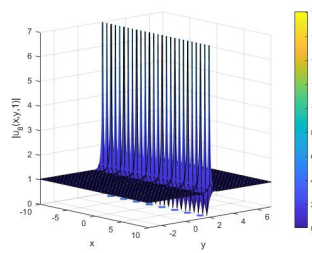

(a) 3D modulus,

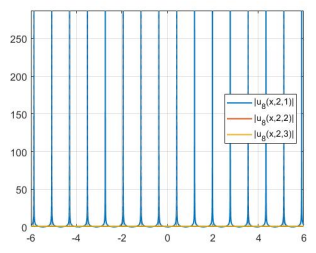

(d) 2D modulus,

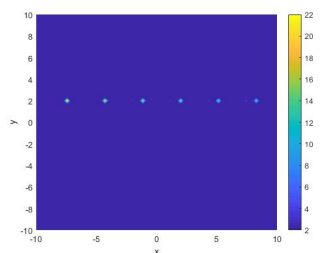

(g) Contour of the modulus,

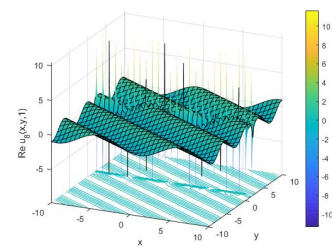

(b) 3D real,

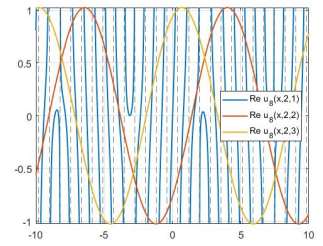

(e) $2 \mathrm{D}$ real,

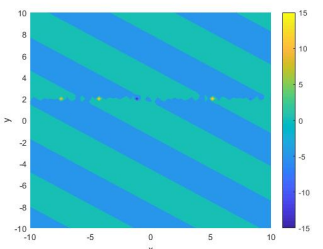

(h) Contour of the real,

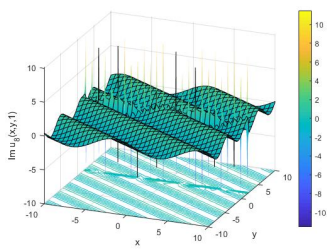

(c) 3D imaginary,

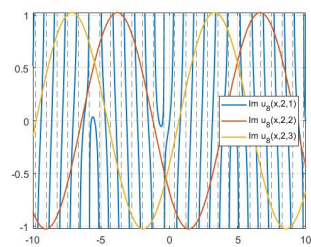

(f) $2 \mathrm{D}$ imaginary,

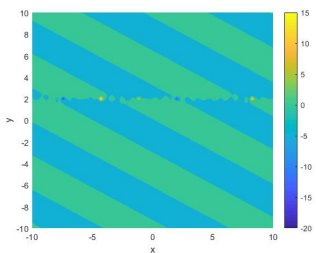

(i) Contour of the imaginary,

Fig. 5 Plots of the solution $u_{12}(x, y, t)$ in Eq. 21 for the parameters $\rho=4, b_{1}=\alpha_{1}=\alpha_{2}=$ $\beta=\lambda_{2}=\varphi=t=1, \lambda_{1}=0.6, \omega=2$, and Set $_{30}$

\section{Conclusion}

In this paper, we have produced new abundant analytic soliton solutions for $(1+2)$ Chiral NLS equation with the aid of the METEM-cNRCS. We have plotted some illustrative figures to show the dynamics of the gained solutions using appropriate parameters. The results of this paper indicate that the technique we used might be an efficient instrument for producing new exact solutions for nonlinear real-life problems in various disciplines.

Acknowledgments. The fourth author (MC) is thankful to TUBITAK (The Scientific and Technological Research Council of Turkey) for the financial support of the 2211-A Fellowship Program.

\section{Declarations.}

Conflict of interest. The authors declare that they have no conflict of interest. 
Data Availability Statements. Data sharing not applicable to this article as no datasets were generated or analysed during the current study.

\section{APPENDIX}

The possible solutions for the system of algebraic equations in Eq. 13 are given in this part.

$$
\begin{aligned}
& \operatorname{Set}_{1}=\left\{\begin{array}{c}
\beta\left(\begin{array}{c}
\left(\left(1 / 4 \alpha_{1}^{2}+1 / 4 \alpha_{2}^{2}\right) \lambda_{2}^{2}+\alpha_{1}^{2}\left(b_{1}^{2}+b_{2}^{2}\right) \rho\right) A_{1}^{4}+ \\
1 / 2 \beta \alpha_{2} \lambda_{2}\left(b_{1}^{2}+b_{2}^{2}\right) A_{1}^{2}+1 / 4 \beta^{2}\left(b_{1}^{2}+b_{2}^{2}\right)^{2}
\end{array}\right) \\
A_{1}^{4} \alpha_{1}^{2} \\
\lambda_{1}=\frac{-4 \frac{A_{1}^{2} \alpha_{2} \lambda_{2}-\beta\left(b_{1}{ }^{2}+b_{2}{ }^{2}\right)}{A_{1}{ }^{2} \alpha_{1}}, A_{0}=0, A_{1}=A_{1}, B_{1}=\rho A_{1}}{}
\end{array}\right\}, \\
& \operatorname{Set}_{2}=\left\{\begin{array}{c}
\beta\left(\begin{array}{c}
\left(\left(-1 / 8 \alpha_{1}{ }^{2}-1 / 8 \alpha_{2}{ }^{2}\right) \lambda_{2}{ }^{2}+\alpha_{1}{ }^{2}\left(b_{1}{ }^{2}+b_{2}{ }^{2}\right) \rho\right) A_{1}{ }^{4}- \\
1 / 4 \beta \alpha_{2} \lambda_{2}\left(b_{1}^{2}+b_{2}^{2}\right) A_{1}^{2}-1 / 8 \beta^{2}\left(b_{1}^{2}+b_{2}^{2}\right)^{2}
\end{array}\right) \\
\omega=8 \frac{A_{1}{ }^{4} \alpha_{1}{ }^{2}}{\lambda_{1}=\frac{-A_{1}{ }^{2} \alpha_{2} \lambda_{2}-\beta\left(b_{1}{ }^{2}+b_{2}{ }^{2}\right)}{A_{1}{ }^{2} \alpha_{1}}, A_{0}=0, A_{1}=A_{1}, B_{1}=-\rho A_{1}} \\
\lambda_{1}
\end{array}\right\}, \\
& \operatorname{Set}_{3}=\left\{\begin{array}{c}
\left(\begin{array}{c}
\left(\left(1 / 4 \alpha_{1}{ }^{2}+1 / 4 \alpha_{2}{ }^{2}\right) \lambda_{1}{ }^{2}+\alpha_{2}{ }^{2}\left(b_{1}{ }^{2}+b_{2}{ }^{2}\right) \rho\right) A_{1}{ }^{4}+ \\
1 / 2 \beta \alpha_{1} \lambda_{1}\left(b_{1}^{2}+b_{2}^{2}\right) A_{1}^{2}+1 / 4 \beta^{2}\left(b_{1}{ }^{2}+b_{2}^{2}\right)^{2}
\end{array}\right) \beta \\
A_{1}^{4} \alpha_{2}{ }^{2} \\
\left.\lambda_{2}=\frac{-4 A_{1}{ }^{2} \alpha_{1} \lambda_{1}-\beta\left(b_{1}{ }^{2}+b_{2}{ }^{2}\right)}{A_{1}{ }^{2} \alpha_{2}}, A_{0}=0, A_{1}=A_{1}, B_{1}=\rho A_{1}\right]
\end{array}\right\}, \\
& \operatorname{Set}_{4}=\left\{\begin{array}{c}
\left(\begin{array}{c}
\left(\left(-1 / 8 \alpha_{1}^{2}-1 / 8 \alpha_{2}^{2}\right) \lambda_{1}^{2}+\alpha_{2}^{2}\left(b_{1}^{2}+b_{2}^{2}\right) \rho\right) A_{1}^{4}- \\
1 / 4 \beta \alpha_{1} \lambda_{1}\left(b_{1}^{2}+b_{2}^{2}\right) A_{1}^{2}-1 / 8 \beta^{2}\left(b_{1}^{2}+b_{2}^{2}\right)^{2}
\end{array}\right) \beta \\
A_{1}^{4} \alpha_{2}^{2} \\
\lambda_{2}=\frac{-A_{1}^{2} \alpha_{1} \lambda_{1}-\beta\left(b_{1}{ }^{2}+b_{2}^{2}\right)}{A_{1}{ }^{2} \alpha_{2}}, A_{0}=0, A_{1}=A_{1}, B_{1}=-\rho A_{1}
\end{array}\right\}, \\
& \text { Set }_{5}=\left\{b_{1}=\frac{1}{\beta} \sqrt{-\beta\left(A_{1}^{2} \alpha_{1} \lambda_{1}+A_{1}^{2} \alpha_{2} \lambda_{2}+\beta b_{2}^{2}\right)}\right. \text {, } \\
& \left.\omega=4 \rho\left(\alpha_{1} \lambda_{1}+\alpha_{2} \lambda_{2}\right){A_{1}}^{2}-\beta{\lambda_{1}}^{2}-\beta{\lambda_{2}}^{2}, A_{0}=0, A_{1}=A_{1}, B_{1}=\rho A_{1}\right\} \\
& \operatorname{Set}_{6}=\left\{b_{1}=-\frac{1}{\beta} \sqrt{-\beta\left(A_{1}^{2} \alpha_{1} \lambda_{1}+A_{1}^{2} \alpha_{2} \lambda_{2}+\beta b_{2}^{2}\right)}\right. \text {, } \\
& \omega=4 \rho\left(\alpha_{1} \lambda_{1}+\alpha_{2} \lambda_{2}\right) A_{1}^{2}-\beta{\lambda_{1}}^{2}-\beta{\lambda_{2}}^{2}, A_{0}=0, \\
& \left.A_{1}=A_{1}, B_{1}=\rho A_{1}\right\} \\
& \text { Set }_{7}=\left\{b_{1}=\frac{1}{\beta} \sqrt{-\beta\left(A_{1}^{2} \alpha_{1} \lambda_{1}+A_{1}^{2} \alpha_{2} \lambda_{2}+\beta b_{2}^{2}\right)}\right. \text {, } \\
& \left.\omega=-8 \rho\left(\alpha_{1} \lambda_{1}+\alpha_{2} \lambda_{2}\right) A_{1}^{2}-\beta \lambda_{1}^{2}-\beta \lambda_{2}^{2}, A_{0}=0, A_{1}=A_{1}, B_{1}=-\rho A_{1}\right\} \\
& \text { Set }_{8}=\left\{b_{1}=-\frac{1}{\beta} \sqrt{-\beta\left(A_{1}^{2} \alpha_{1} \lambda_{1}+A_{1}^{2} \alpha_{2} \lambda_{2}+\beta b_{2}^{2}\right)}\right. \text {, } \\
& \left.\omega=-8 \rho\left(\alpha_{1} \lambda_{1}+\alpha_{2} \lambda_{2}\right) A_{1}{ }^{2}-\beta{\lambda_{1}}^{2}-\beta{\lambda_{2}}^{2}, A_{0}=0, A_{1}=A_{1}, B_{1}=-\rho A_{1}\right\} \\
& \operatorname{Set}_{9}=\left\{b_{2}=\frac{1}{\beta} \sqrt{-\beta\left(A_{1}^{2} \alpha_{1} \lambda_{1}+A_{1}^{2} \alpha_{2} \lambda_{2}+\beta b_{1}^{2}\right)},\right. \\
& \left.\omega=4 \rho\left(\alpha_{1} \lambda_{1}+\alpha_{2} \lambda_{2}\right) A_{1}^{2}-\beta{\lambda_{1}}^{2}-\beta{\lambda_{2}}^{2}, A_{0}=0, A_{1}=A_{1}, B_{1}=\rho A_{1}\right\}
\end{aligned}
$$




$$
\begin{aligned}
& \operatorname{Set}_{10}=\left\{b_{2}=-\frac{1}{\beta} \sqrt{-\beta\left(A_{1}^{2} \alpha_{1} \lambda_{1}+A_{1}^{2} \alpha_{2} \lambda_{2}+\beta b_{1}^{2}\right)},\right. \\
& \left.\omega=4 \rho\left(\alpha_{1} \lambda_{1}+\alpha_{2} \lambda_{2}\right) A_{1}{ }^{2}-\beta{\lambda_{1}}^{2}-\beta{\lambda_{2}}^{2}, A_{0}=0, A_{1}=A_{1}, B_{1}=\rho A_{1}\right\} \\
& \operatorname{Set}_{11}=\left\{b_{2}=\frac{1}{\beta} \sqrt{-\beta\left(A_{1}^{2} \alpha_{1} \lambda_{1}+A_{1}^{2} \alpha_{2} \lambda_{2}+\beta b_{1}^{2}\right)},\right. \\
& \omega=-8 \rho\left(\alpha_{1} \lambda_{1}+\alpha_{2} \lambda_{2}\right) A_{1}^{2}-\beta{\lambda_{1}}^{2}-\beta{\lambda_{2}}^{2}, A_{0}=0, \\
& \left.A_{1}=A_{1}, B_{1}=-\rho A_{1}\right\} \\
& \operatorname{Set}_{12}=\left\{b_{2}=-\frac{1}{\beta} \sqrt{-\beta\left(A_{1}^{2} \alpha_{1} \lambda_{1}+A_{1}^{2} \alpha_{2} \lambda_{2}+\beta b_{1}^{2}\right)},\right.
\end{aligned}
$$

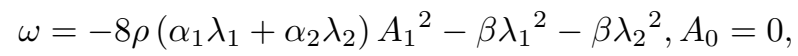

$$
\begin{aligned}
& \left.A_{1}=A_{1}, B_{1}=-\rho A_{1}\right\}
\end{aligned}
$$

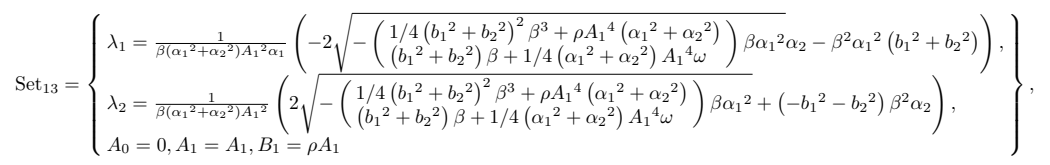

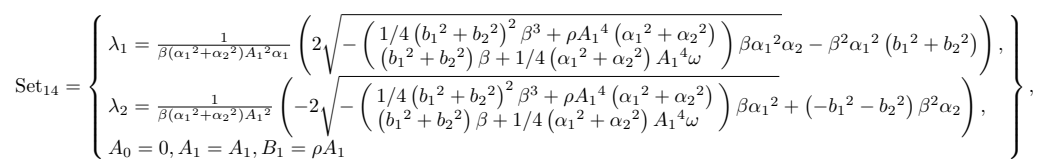

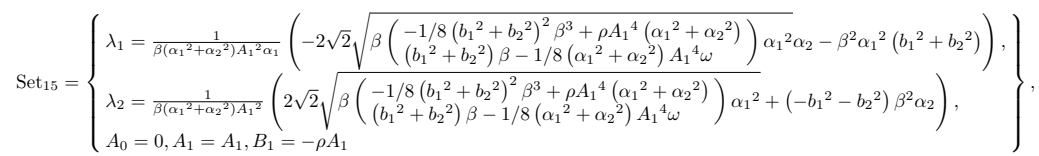

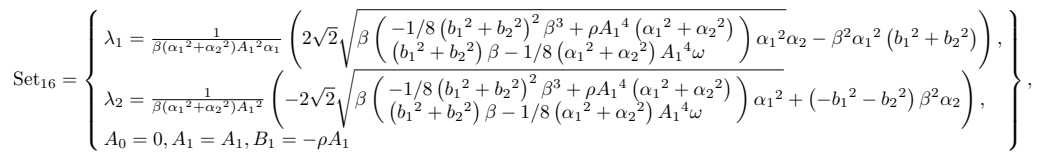

$$
\begin{aligned}
& \operatorname{Set}_{17}=\left\{\begin{array}{l}
\omega=-\left(4 \rho b_{1}^{2}+4 \rho b_{2}^{2}+\lambda_{1}^{2}+\lambda_{2}^{2}\right) \beta, A_{0}=0, A_{1}=\frac{1}{\alpha_{1} \lambda_{1}+\alpha_{2} \lambda_{2}} \sqrt{-\left(\alpha_{1} \lambda_{1}+\alpha_{2} \lambda_{2}\right) \beta\left(b_{1}{ }^{2}+b_{2}{ }^{2}\right)}, \\
B_{1}=\frac{\rho}{\alpha_{1} \lambda_{1}+\alpha_{2} \lambda_{2}} \sqrt{-\left(\alpha_{1} \lambda_{1}+\alpha_{2} \lambda_{2}\right) \beta\left(b_{1}{ }^{2}+b_{2}{ }^{2}\right)}
\end{array}\right\}, \\
& \operatorname{Set}_{18}=\left\{\begin{array}{l}
\omega=\left(8 \rho b_{1}{ }^{2}+8 \rho b_{2}{ }^{2}-\lambda_{1}{ }^{2}-\lambda_{2}{ }^{2}\right) \beta, A_{0}=0, A_{1}=\frac{1}{\alpha_{1} \lambda_{1}+\alpha_{2} \lambda_{2}} \sqrt{-\left(\alpha_{1} \lambda_{1}+\alpha_{2} \lambda_{2}\right) \beta\left(b_{1}{ }^{2}+b_{2}{ }^{2}\right)}, \\
B_{1}=-\frac{\rho}{\alpha_{1} \lambda_{1}+\alpha_{2} \lambda_{2}} \sqrt{-\left(\alpha_{1} \lambda_{1}+\alpha_{2} \lambda_{2}\right) \beta\left(b_{1}{ }^{2}+b_{2}{ }^{2}\right)}
\end{array}\right\}, \\
& \operatorname{Set}_{19}=\left\{\begin{array}{l}
\omega=\left(8 \rho b_{1}{ }^{2}+8 \rho b_{2}{ }^{2}-\lambda_{1}{ }^{2}-\lambda_{2}{ }^{2}\right) \beta, A_{0}=0, A_{1}=-\frac{1}{\alpha_{1} \lambda_{1}+\alpha_{2} \lambda_{2}} \sqrt{-\left(\alpha_{1} \lambda_{1}+\alpha_{2} \lambda_{2}\right) \beta\left(b_{1}{ }^{2}+b_{2}{ }^{2}\right)}, \\
B_{1}=\frac{\rho}{\alpha_{1} \lambda_{1}+\alpha_{2} \lambda_{2}} \sqrt{-\left(\alpha_{1} \lambda_{1}+\alpha_{2} \lambda_{2}\right) \beta\left(b_{1}{ }^{2}+b_{2}{ }^{2}\right)}
\end{array}\right\}, \\
& \operatorname{Set}_{20}=\left\{\begin{array}{l}
\omega=-\left(4 \rho b_{1}{ }^{2}+4 \rho b_{2}{ }^{2}+\lambda_{1}^{2}+\lambda_{2}{ }^{2}\right) \beta, A_{0}=0, A_{1}=-\frac{1}{\alpha_{1} \lambda_{1}+\alpha_{2} \lambda_{2}} \sqrt{-\left(\alpha_{1} \lambda_{1}+\alpha_{2} \lambda_{2}\right) \beta\left(b_{1}{ }^{2}+b_{2}{ }^{2}\right)}, \\
B_{1}=-\frac{\rho}{\alpha_{1} \lambda_{1}+\alpha_{2} \lambda_{2}} \sqrt{-\left(\alpha_{1} \lambda_{1}+\alpha_{2} \lambda_{2}\right) \beta\left(b_{1}{ }^{2}+b_{2}{ }^{2}\right)}
\end{array}\right\}, \\
& \operatorname{Set}_{21}=\left\{\begin{array}{l}
b_{1}=\frac{\sqrt{2}}{4 \beta \rho} \sqrt{-\beta \rho\left(8 \beta \rho b_{2}^{2}-\beta \lambda_{1}^{2}-\beta \lambda_{2}^{2}-\omega\right)}, A_{0}=0, A_{1}=\frac{\sqrt{2}}{4\left(\alpha_{1} \lambda_{1}+\alpha_{2} \lambda_{2}\right) \rho} \sqrt{\frac{-\left(\alpha_{1} \lambda_{1}+\alpha_{2} \lambda_{2}\right)}{\rho\left(\left(\lambda_{1}^{2}+\lambda_{2}^{2}\right) \beta+\omega\right)},} \\
B_{1}=\frac{\left(\left(\lambda_{1}^{2}+\lambda_{2}^{2}\right) \beta+\omega\right) \rho \sqrt{2}}{4} \frac{1}{\sqrt{-\left(\alpha_{1} \lambda_{1}+\alpha_{2} \lambda_{2}\right) \rho\left(\left(\lambda_{1}{ }^{2}+\lambda_{2}{ }^{2}\right) \beta+\omega\right)}}
\end{array}\right\}, \\
& \operatorname{Set}_{22}=\left\{\begin{array}{l}
b_{1}=-\frac{\sqrt{2}}{4 \beta \rho} \sqrt{-\beta \rho\left(8 \beta \rho b_{2}{ }^{2}-\beta \lambda_{1}{ }^{2}-\beta \lambda_{2}{ }^{2}-\omega\right)}, A_{0}=0, A_{1}=\frac{\sqrt{2}}{4\left(\alpha_{1} \lambda_{1}+\alpha_{2} \lambda_{2}\right) \rho} \sqrt{\frac{-\left(\alpha_{1} \lambda_{1}+\alpha_{2} \lambda_{2}\right) \rho}{\left(\left(\lambda_{1}{ }^{2}+\lambda_{2}{ }^{2}\right) \beta+\omega\right)},} \\
B_{1}=\frac{\left(\left(\lambda_{1}{ }^{2}+\lambda_{2}{ }^{2}\right) \beta+\omega\right) \rho \sqrt{2}}{4} \frac{1}{\sqrt{-\left(\alpha_{1} \lambda_{1}+\alpha_{2} \lambda_{2}\right) \rho\left(\left(\lambda_{1}{ }^{2}+\lambda_{2}{ }^{2}\right) \beta+\omega\right)}}
\end{array}\right\}, \\
& \operatorname{Set}_{23}=\left\{\begin{array}{l}
b_{1}=\frac{\sqrt{2}}{4 \beta \rho} \sqrt{-\beta \rho\left(8 \beta \rho b_{2}{ }^{2}-\beta \lambda_{1}{ }^{2}-\beta \lambda_{2}{ }^{2}-\omega\right)}, A_{0}=0, A_{1}=-\frac{\sqrt{2}}{4\left(\alpha_{1} \lambda_{1}+\alpha_{2} \lambda_{2}\right) \rho} \sqrt{\frac{-\left(\alpha_{1} \lambda_{1}+\alpha_{2} \lambda_{2}\right) \rho}{\left(\left(\lambda_{1}{ }^{2}+\lambda_{2}{ }^{2}\right) \beta+\omega\right)},} \\
B_{1}=-\frac{\left(\left(\lambda_{1}{ }^{2}+\lambda_{2}{ }^{2}\right) \beta+\omega\right) \rho \sqrt{2}}{4} \frac{1}{\sqrt{-\left(\alpha_{1} \lambda_{1}+\alpha_{2} \lambda_{2}\right) \rho\left(\left(\lambda_{1}{ }^{2}+\lambda_{2}{ }^{2}\right) \beta+\omega\right)}}
\end{array}\right\},
\end{aligned}
$$


$\operatorname{Set}_{24}=\left\{\begin{array}{l}b_{1}=-\frac{\sqrt{2}}{4 \beta \rho} \sqrt{-\beta \rho\left(8 \beta \rho b_{2}{ }^{2}-\beta \lambda_{1}{ }^{2}-\beta \lambda_{2}{ }^{2}-\omega\right)}, A_{0}=0, A_{1}=-\frac{\sqrt{2}}{4\left(\alpha_{1} \lambda_{1}+\alpha_{2} \lambda_{2}\right) \rho} \sqrt{-\left(\alpha_{1} \lambda_{1}+\alpha_{2} \lambda_{2}\right) \rho} \\ \left(\left(\lambda_{1}{ }^{2}+\lambda_{2}{ }^{2}\right) \beta+\omega\right)\end{array},\right\}$,

$\operatorname{Set}_{25}=\left\{\begin{array}{l}b_{1}=\frac{1}{2 \beta \rho} \sqrt{-\beta \rho\left(4 \beta \rho b_{2}^{2}+\beta \lambda_{1}^{2}+\beta \lambda_{2}^{2}+\omega\right)}, A_{0}=0, A_{1}=\frac{1}{2\left(\alpha_{1} \lambda_{1}+\alpha_{2} \lambda_{2}\right) \rho} \sqrt{\begin{array}{l}\left(\alpha_{1} \lambda_{1}+\alpha_{2} \lambda_{2}\right) \rho \\ \left.\left(\lambda_{1}^{2}+\lambda_{2}^{2}\right) \beta+\omega\right)\end{array}} \\ B_{1}=\frac{\left(\left(\lambda_{1}^{2}+\lambda_{2}^{2}\right) \beta+\omega\right) \rho}{2} \frac{1}{\sqrt{\left(\alpha_{1} \lambda_{1}+\alpha_{2} \lambda_{2}\right) \rho\left(\left(\lambda_{1}{ }^{2}+\lambda_{2}{ }^{2}\right) \beta+\omega\right)}}\end{array}\right\}$,

$\operatorname{Set}_{26}=\left\{\begin{array}{l}b_{1}=-\frac{1}{2 \beta \rho} \sqrt{-\beta \rho\left(4 \beta \rho b_{2}{ }^{2}+\beta \lambda_{1}{ }^{2}+\beta \lambda_{2}{ }^{2}+\omega\right)}, A_{0}=0, A_{1}=\frac{1}{2\left(\alpha_{1} \lambda_{1}+\alpha_{2} \lambda_{2}\right) \rho} \sqrt{\begin{array}{l}\left(\alpha_{1} \lambda_{1}+\alpha_{2} \lambda_{2}\right) \rho \\ \left(\left(\lambda_{1}{ }^{2}+\lambda_{2}{ }^{2}\right) \beta+\omega\right)\end{array}}, \\ B_{1}=\frac{\left(\left(\lambda_{1}{ }^{2}+\lambda_{2}{ }^{2}\right) \beta+\omega\right) \rho}{2} \frac{1}{\sqrt{\left(\alpha_{1} \lambda_{1}+\alpha_{2} \lambda_{2}\right) \rho\left(\left(\lambda_{1}{ }^{2}+\lambda_{2}{ }^{2}\right) \beta+\omega\right)}}\end{array}\right\}$,

$\operatorname{Set}_{27}=\left\{\begin{array}{l}b_{1}=\frac{1}{2 \beta \rho} \sqrt{-\beta \rho\left(4 \beta \rho b_{2}{ }^{2}+\beta \lambda_{1}^{2}+\beta \lambda_{2}{ }^{2}+\omega\right)}, A_{0}=0, A_{1}=-\frac{1}{2\left(\alpha_{1} \lambda_{1}+\alpha_{2} \lambda_{2}\right) \rho} \sqrt{\begin{array}{l}\left(\alpha_{1} \lambda_{1}+\alpha_{2} \lambda_{2}\right) \rho \\ \left(\left(\lambda_{1}{ }^{2}+\lambda_{2}{ }^{2}\right) \beta+\omega\right)\end{array}} \\ B_{1}=-\frac{\left(\left(\lambda_{1}{ }^{2}+\lambda_{2}{ }^{2}\right) \beta+\omega\right) \rho}{2} \frac{1}{\sqrt{\left(\alpha_{1} \lambda_{1}+\alpha_{2} \lambda_{2}\right) \rho\left(\left(\lambda_{1}{ }^{2}+\lambda_{2}{ }^{2}\right) \beta+\omega\right)}}\end{array}\right\}$,

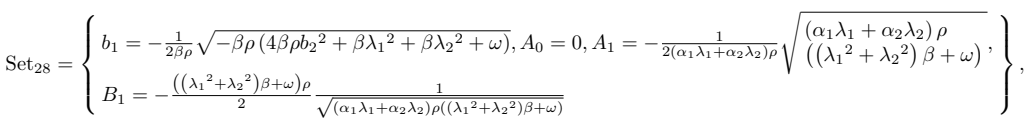

$\operatorname{Set}_{29}=\left\{\begin{array}{l}b_{2}=\frac{\sqrt{2}}{4 \beta \rho} \sqrt{-\beta \rho\left(8 \beta \rho b_{1}{ }^{2}-\beta \lambda_{1}{ }^{2}-\beta \lambda_{2}{ }^{2}-\omega\right)}, A_{0}=0, A_{1}=\frac{\sqrt{2}}{4\left(\alpha_{1} \lambda_{1}+\alpha_{2} \lambda_{2}\right) \rho} \sqrt{\frac{-\left(\alpha_{1} \lambda_{1}+\alpha_{2} \lambda_{2}\right) \rho}{\left(\left(\lambda_{1}{ }^{2}+\lambda_{2}{ }^{2}\right) \beta+\omega\right)},} \\ B_{1}=\frac{\left(\left(\lambda_{1}{ }^{2}+\lambda_{2}{ }^{2}\right) \beta+\omega\right) \rho \sqrt{2}}{4} \frac{1}{\sqrt{-\left(\alpha_{1} \lambda_{1}+\alpha_{2} \lambda_{2}\right) \rho\left(\left(\lambda_{1}{ }^{2}+\lambda_{2}{ }^{2}\right) \beta+\omega\right)}}\end{array}\right\}$,

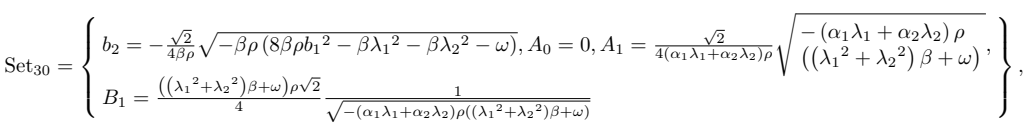

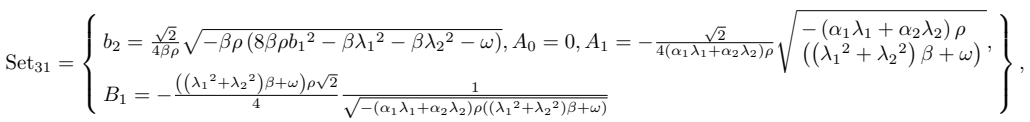

$\operatorname{Set}_{32}=\left\{\begin{array}{l}b_{2}=-\frac{\sqrt{2}}{4 \beta \rho} \sqrt{-\beta \rho\left(8 \beta \rho b_{1}^{2}-\beta \lambda_{1}{ }^{2}-\beta \lambda_{2}{ }^{2}-\omega\right)}, A_{0}=0, A_{1}=-\frac{\sqrt{2}}{4\left(\alpha_{1} \lambda_{1}+\alpha_{2} \lambda_{2}\right) \rho} \sqrt{\frac{-\left(\alpha_{1} \lambda_{1}+\alpha_{2} \lambda_{2}\right) \rho}{\left(\left(\lambda_{1}^{2}+\lambda_{2}^{2}\right) \beta+\omega\right)},} \\ B_{1}=-\frac{\left(\left(\lambda_{1}{ }^{2}+\lambda_{2}{ }^{2}\right) \beta+\omega\right) \rho \sqrt{2}}{4} \frac{1}{\sqrt{-\left(\alpha_{1} \lambda_{1}+\alpha_{2} \lambda_{2}\right) \rho\left(\left(\lambda_{1}{ }^{2}+\lambda_{2}{ }^{2}\right) \beta+\omega\right.}}\end{array}\right\}$,

$\operatorname{Set}_{33}=\left\{\begin{array}{l}b_{2}=\frac{1}{2 \beta} \sqrt{-\beta \rho\left(4 \beta \rho b_{1}{ }^{2}+\beta \lambda_{1}{ }^{2}+\beta \lambda_{2}{ }^{2}+\omega\right)}, A_{0}=0, A_{1}=\frac{1}{2\left(\alpha_{1} \lambda_{1}+\alpha_{2} \lambda_{2}\right) \rho} \sqrt{\left(\begin{array}{l}\left(\alpha_{1} \lambda_{1}+\alpha_{2} \lambda_{2}\right) \rho \\ \left(\left(\lambda_{1}{ }^{2}+\lambda_{2}{ }^{2}\right) \beta+\omega\right.\end{array}\right)} \\ B_{1}=\frac{\left(\left(\lambda_{1}{ }^{2}+\lambda_{2}{ }^{2}\right) \beta+\omega\right) \rho}{2} \frac{1}{\sqrt{\left(\alpha_{1} \lambda_{1}+\alpha_{2} \lambda_{2}\right) \rho\left(\left(\lambda_{1}{ }^{2}+\lambda_{2}{ }^{2}\right) \beta+\omega\right)}}\end{array}\right\}$,

$\operatorname{Set}_{34}=\left\{\begin{array}{l}b_{2}=-\frac{1}{2 \beta \rho} \sqrt{-\beta \rho\left(4 \beta \rho b_{1}^{2}+\beta \lambda_{1}^{2}+\beta \lambda_{2}^{2}+\omega\right)}, A_{0}=0, A_{1}=\frac{1}{2\left(\alpha_{1} \lambda_{1}+\alpha_{2} \lambda_{2}\right) \rho} \sqrt{\left(\begin{array}{l}\left(\alpha_{1} \lambda_{1}+\alpha_{2} \lambda_{2}\right) \rho \\ \left(\left(\lambda_{1}^{2}+\lambda_{2}^{2}\right) \beta+\omega\right)\end{array}\right.} \\ B_{1}=\frac{\left(\left(\lambda_{1}^{2}+\lambda_{2}^{2}\right) \beta+\omega\right) \rho}{2} \frac{1}{\sqrt{\left.\left(\alpha_{1} \lambda_{1}+\alpha_{2} \lambda_{2}\right) \rho\left(\left(\lambda_{1}^{2}+\lambda_{2}\right)^{2}\right) \beta+\omega\right)}}\end{array}\right\}$,

$\operatorname{Set}_{35}=\left\{\begin{array}{l}b_{2}=\frac{1}{2 \beta \rho} \sqrt{-\beta \rho\left(4 \beta \rho b_{1}{ }^{2}+\beta \lambda_{1}{ }^{2}+\beta \lambda_{2}{ }^{2}+\omega\right)}, A_{0}=0, A_{1}=-\frac{1}{2\left(\alpha_{1} \lambda_{1}+\alpha_{2} \lambda_{2}\right) \rho} \sqrt{\left(\begin{array}{l}\left(\alpha_{1} \lambda_{1}+\alpha_{2} \lambda_{2}\right) \rho \\ \left(\left(\lambda_{1}^{2}+\lambda_{2}^{2}\right) \beta+\omega\right)\end{array}\right.} \\ B_{1}=-\frac{\left(\left(\lambda_{1}^{2}+\lambda_{2}^{2}\right) \beta+\omega\right) \rho}{2)} \frac{1}{\sqrt{\left.\left(\alpha_{1} \lambda_{1}+\alpha_{2} \lambda_{2}\right) \rho\left(\lambda_{1}{ }^{2}+\lambda_{2}{ }^{2}\right) \beta+\omega\right)}}\end{array}\right\}$,

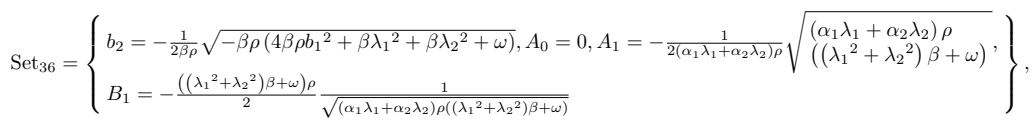




\section{References}

[1] Zhou, Q., Liu, L., Zhang, H., Wei, C., Lu, J., Yu, H., Biswas, A.: Analytical study of Thirring optical solitons with parabolic law nonlinearity and spatio-temporal dispersion. Eur. Phys. J. Plus 130(7), 1-6 (2015). https: //doi.org/10.1140/epjp/i2015-15138-9

[2] Cinar, M., Onder, I., Secer, A., Yusuf, A., Sulaiman, T.A., Bayram, M., Aydin, H.: Soliton Solutions of $(2+1)$ Dimensional Heisenberg Ferromagnetic Spin Equation by the Extended Rational sine-cosine and sinh-cosh Method. Int. J. Appl. Comput. Math. 7(4), 1-17 (2021). https: //doi.org/10.1007/s40819-021-01076-5

[3] Zhou, Q., Zhu, Q., Liu, Y., Yu, H., Yao, P., Biswas, A.: Thirring optical solitons in birefringent fibers with spatio-temporal dispersion and Kerr law nonlinearity. Laser Phys. 25(1), 015402 (2014). https://doi.org/10. 1088/1054-660x/25/1/015402

[4] Zhou, Q., Mirzazadeh, M., Zerrad, E., Biswas, A., Belic, M.: Bright, dark, and singular solitons in optical fibers with spatio-temporal dispersion and spatially dependent coefficients. J. Mod. Opt. 63(10), 950-954 (2016). https://doi.org/10.1080/09500340.2015.1111456

[5] Secer, A., Cinar, M.: A Jacobi wavelet collocation method for fractional fisher's equation in time. Thermal Science 24(Suppl. 1), 119-129 (2020)

[6] Liu, X., Triki, H., Zhou, Q., Liu, W., Biswas, A.: Analytic study on interactions between periodic solitons with controllable parameters. Nonlinear Dynamics 94(1), 703-709 (2018). https://doi.org/10.1007/ s11071-018-4387-7

[7] Cinar, M., Secer, A., Bayram, M.: An application of Genocchi wavelets for solving the fractional Rosenau-Hyman equation. Alexandria Engineering Journal 60(6), 5331-5340 (2021). https://doi.org/10.1016/j.aej.2021.04. 037

[8] Ozdemir, N., Esen, H., Secer, A., Bayram, M., Yusuf, A., Sulaiman, T.A.: Optical Soliton Solutions to Chen Lee Liu model by the modified extended tanh expansion scheme. Optik 245, 167643 (2021). https://doi.org/10. 1016/j.ijleo.2021.167643

[9] Cinar, M., Onder, I., Secer, A., Sulaiman, T.A., Yusuf, A., Bayram, M.: Optical solitons of the $(2+1)$-dimensional Biswas-Milovic equation using modified extended tanh-function method. Optik 245, 167631 (2021). https://doi.org/10.1016/j.ijleo.2021.167631

[10] Esen, H., Ozdemir, N., Secer, A., Bayram, M., Sulaiman, T.A., Yusuf, 
A.: Solitary wave solutions of chiral nonlinear Schrödinger equations. Mod. Phys. Lett. B 35(30), 2150472 (2021). https://doi.org/10.1142/ S0217984921504728

[11] Cinar, M., Onder, I., Secer, A., Yusuf, A., Sulaiman, T.A., Bayram, M., Aydin, H.: The analytical solutions of Zoomeron equation via extended rational sin-cos and sinh-cosh methods. Phys. Scr. 96(9), 094002 (2021). https://doi.org/10.1088/1402-4896/ac0374

[12] Cazenave, T., Weissler, F.B.: The cauchy problem for the critical nonlinear Schrödinger equation in Hs. Nonlinear Analysis 14(10), 807-836 (1990). https://doi.org/10.1016/0362-546X(90)90023-A

[13] Geng, X., Lv, Y.: Darboux transformation for an integrable generalization of the nonlinear Schrödinger equation. Nonlinear Dynamics 69(4), 16211630 (2012). https://doi.org/10.1007/s11071-012-0373-7

[14] Lebowitz, J.L., Rose, H.A., Speer, E.R.: Statistical mechanics of the nonlinear Schrödinger equation. Journal of Statistical Physics 50(3-4), 657-687 (1988). https://doi.org/10.1007/BF01026495

[15] Besse, C.: A relaxation scheme for the nonlinear schrödinger equation. SIAM Journal on Numerical Analysis 42(3), 934-952 (2004). https://doi. org/10.1137/S0036142901396521

[16] Longuet-Higgins, M.S.: Note on a modification to the nonlinear Schrödinger equation for application to deep water waves. Proceedings of the Royal Society of London. A. Mathematical and Physical Sciences 369(1736), 105-114 (1979). https://doi.org/10.1098/rspa.1979.0154

[17] Sulaiman, T.A., Yusuf, A., Abdel-Khalek, S., Bayram, M., Ahmad, H.: Nonautonomous complex wave solutions to the $(2+1)$-dimensional variable-coefficients nonlinear Chiral Schrödinger equation. Results in Physics 19, 103604 (2020). https://doi.org/10.1016/j.rinp.2020.103604

[18] Zhang, X., Chen, Y.: Inverse scattering transformation for generalized nonlinear Schrödinger equation. Applied Mathematics Letters 98, 306313 (2019). https://doi.org/10.1016/j.aml.2019.06.014

[19] Zhou, Q., Zhu, Q., Liu, Y., Yu, H., Yao, P., Biswas, A.: Thirring optical solitons in birefringent bers with spatio-temporal dispersion and Kerr law nonlinearity. Laser Physics 25(1), 015402 (2015). https://doi.org/10. 1088/1054-660X/25/1/015402

[20] Jiang, H.-J., Xiang, J.-J., Dai, C.-Q., Wang, Y.-Y.: Nonautonomous bright soliton solutions on continuous wave and cnoidal wave backgrounds in blood vessels. Nonlinear Dynamics 75(1), 201-207 (2014). https://doi. 
org/10.1007/s11071-013-1058-6

[21] Korkmaz, A., Dağ, İ.: A differential quadrature algorithm for nonlinear Schrödinger equation. Nonlinear Dynamics 56(1), 69-83 (2009). https: //doi.org/10.1007/s11071-008-9380-0

[22] Seadawy, A.R.: Modulation instability analysis for the generalized derivative higher order nonlinear Schrödinger equation and its the bright and dark soliton solutions. Journal of Electromagnetic Waves and Applications 31(14), 1353-1362 (2017). https://doi.org/10.1080/09205071.2017. 1348262

[23] Alshahrani, B., Yakout, H.A., Khater, M.M.A., Abdel-Aty, A.-H., Mahmoud, E.E., Baleanu, D., Eleuch, H.: Accurate novel explicit complex wave solutions of the $(2+1)$-dimensional Chiral nonlinear Schrödinger equation. Results Phys. 23, 104019 (2021). https://doi.org/10.1016/j. rinp.2021.104019

[24] Biswas, A.: Chiral Solitons in 1+2 Dimensions. Int. J. Theor. Phys. 48(12), 3403-3409 (2009). https://doi.org/10.1007/s10773-009-0145-4

[25] Hosseini, K., Mirzazadeh, M.: Soliton and other solutions to the $(1+$ 2)-dimensional chiral nonlinear Schrödinger equation. Commun. Theor. Phys. 72(12), 125008 (2020). https://doi.org/10.1088/1572-9494/abb87b

[26] Eslami, M.: Trial solution technique to chiral nonlinear Schrodinger's equation in $(1+2)$-dimensions. Nonlinear Dynamics 85(2), 813-816 (2016). https://doi.org/10.1007/s11071-016-2724-2

[27] Nishino, A., Umeno, Y., Wadati, M.: Chiral nonlinear Schrödinger equation. Chaos, Solitons Fractals 9(7), 1063-1069 (1998). https://doi. org/10.1016/S0960-0779(97)00184-7

[28] Ozisik, M.: On the optical soliton solution of the $(1+1)$ - dimensional perturbed NLSE in optical nano-fibers. Optik 250, 168233 (2022). https: //doi.org/10.1016/j.ijleo.2021.168233

[29] Tang, X., Cai, G., Wu, L.: Hyperbolic function solutions to the $(3+1)$ dimensional Burgers System. World Journal of Modelling and Simulation 4, 278-286 (2009) 